\title{
Mapeo científico de las adicciones comportamentales en el caso de las revistas peruanas
}

\author{
Edwin Salas-Blas ${ }^{1}$; Anthony Copez-Lonzoy*2; Victoria Yance-Yupari ${ }^{3}$ \\ ${ }^{1}$ https://orcid.org/0000-0002-0625-0313, Universidad de San Martín de Porres, Lima, Perú, ${ }^{2}$ https://orcid.org/0000-0003-4761- \\ 4272, Universidad San Ignacio de Loyola, Lima, Perú, Instituto Peruano de Orientación Psicológica, Lima, Perú. Asociación \\ Peruana de Profesionales de las Adicciones - APPADIC, Lima, Perú. Instituto de Investigación, Capacitación y Desarrollo Psicosocial \\ y Educativo - PsycoPerú, Lima, Perú. ${ }^{3 h t t p s: / / o r c i d . o r g / 0000-0002-5089-3957, ~ U n i v e r s i d a d ~ d e ~ S a n ~ M a r t i ́ n ~ d e ~ P o r r e s, ~ L i m a, ~ P e r u ́ . ~}$ \\ ${ }^{*}$ *Los autores Salas-Blas y Copez-Lonzoy solicitan el reconocimiento de la doble primera autoría.
}

Citar como: Salas-Blas, E., Copez-Lonzoy, A., \& Yance-Yupari, V. (2021). Mapeo científico de las adicciones comportamentales en el caso de las revistas peruanas. Revista Digital de Investigación en Docencia Universitaria, 15(2), e1460. https://doi. org/10.19083/10.19083/ridu.2021.1460

Recibido: 30/03/2021. Revisado: 11/06/2021. Aceptado: 08/07/2021. Publicado: 26/11/2021.

\section{Resumen}

Introducción: Las publicaciones sobre conductas adictivas han ido en aumento incluyendo su heterogeneidad contextual en diferentes plataformas virtuales con el fin de generar visibilidad. Objetivo: Identificar las redes de colaboración, autoría y palabras clave sobre conductas adictivas. Método: Se utilizó el mapeo científico e indicadores bibliométricos para analizar la evolución de las conductas adictivas en revistas indizadas y no indizadas de Perú. Resultados: 38\% de artículos fueron de autoría única, 43 de ellos usaron datos cuantitativos (81\%) 23 trabajaron con participantes jóvenes (53.5\%), 37 son estudios empíricos (69.9\%) y la adicción a internet fue más frecuente (39.6\%). Las revistas peruanas en SCI a partir del 2006 han presentado por lo menos un valor de índice $\mathrm{h}=1$. Conclusión: Se evidencia dificultad para rastrear estudios sobre conductas adictivas en revistas peruanas que, sumado a la falta de normativas claras, generan una tarea compleja que influye en su falta de visibilidad.

Palabras clave: adicción; publicación científica; bases de datos; indización.

\section{Scientific mapping of behavioral addictions in the case of peruvian journals}

\begin{abstract}
Background: Publications on addictive behaviors have been increasing including their contextual heterogeneity in different virtual platforms in order to generate visibility. Objective: it was to identify the networks of collaboration, authorship and keywords on addictive behaviors. Method: Scientific mapping and bibliometric indicators were used to analyze the evolution of addictive behaviors in indexed and non-indexed journals in Peru. Results: $38 \%$ of articles were single authorship, 43 of them use quantitative data (81\%), 23 worked with young participants (53.5\%), 37 are empirical studies (69.9\%) and internet addiction was more frequent (39.6\%). Peruvian journals in SCI since 2006 have presented at least an index value $\mathrm{h}=1$. Conclusion: there is evidence of difficulty in tracking studies on addictive behaviors in Peruvian journals, added to the lack of clear regulations, generate a complex task that influences its lack of visibility.
\end{abstract}

Keywords: addiction; scientific publication; databases; indexing. 


\section{Introducción}

Las conductas adictivas han ido en aumento en las últimas dos décadas (Oficina de las Naciones Unidas Contra la Droga y el Delito [UNODC], 2019) y han tomado diferentes rutas y accesos, generando consecuencias perjudiciales en diferentes poblaciones, así como diversos modelos explicativos para su abordaje (Andreassen, 2015; Apud \& Romaní, 2016). Dentro de los accesos a este tipo de conductas, se diferencian dos grandes campos: las conductas adictivas relacionadas con sustancias (convencionales) y las adicciones sin sustancias o comportamentales (Braun et al., 2019; Cía, 2014; Echeburúa, 2016; Hormes et al., 2014; Kuss et al., 2017; Van Rooij \& Prause, 2014). Esta diferencia en el tipo de acceso y comportamiento adictivo no implica una menor atención, debido a que este segundo bloque (adicciones sin sustancias) también evidencia una dinámica peligrosa en el deterioro de diferentes campos en el ámbito personal, social y familiar (Becoña \& Oblitas, 2003; Bisso-Andrade, 2013; Chávez et al., 2017); configurando una ruta con el estudio de ludopatías, adicción a redes sociales, uso problemático de smarthphone, apuestas en línea, cybersexo e incluso el trabajo (Cotlear et al., 2009; Estévez et al., 2017; Goldberg, 1995; Lara \& Miyahira, 2009; Leon, 2014; Marín, 2018; Palomares, 2017; Rengifo et al., 2015; Salas-Blas \& Escurra, 2014; Salas-Blas et al., 2017; Salas-Blas \& Copez-Lonzoy, 2018; Yarlequé et al., 2013).

Sin embargo, algunas de las consecuencias de las adicciones sin sustancias no pueden observarse con rapidez debido a que inicialmente los patrones comportamentales no son diferentes de lo que sucede normalmente, en todo caso se puede afirmar que el deterioro se percibe con cierta lentitud y cuando se manifiesta conjuntamente con otras consecuencias (estudios, al trabajo, a la familia y los amigos, etc.); a pesar de que numerosos estudios advierten las implicancias en la relación con factores psicopatológicos (Araujo-Robles, 2015, 2016a, 2016b; Casale \& Fioraventi, 2018; Castro \& Mahamud, 2017; Castro, et al., 2012; Cruzado et al., 2006; Donelly \& Kuss, 2016; Echeburúa, 2012; Han et al., 2018; Rojas-Jara et al., 2020; Yen et al., 2019; Yu \& Cho, 2016), con conductas disruptivas (Cañón et al., 2016; Castaño \&
Paez, 2019, Matalinares et al., 2013a, 2013b), problemas con la ley (Dhaha, 2013), asociación con abuso de sustancias (Cruzado et al., 2006; Rücker et al., 2015), bases de datos secundaria (Arcaya et al., 2012) y personalidad (Arias \& Masías, 2016).

En ese sentido, la divulgación del conocimiento a través de las publicaciones realizadas en este campo específico, es importante para tener en cuenta la evolución del constructo a través del tiempo (Perestelo-Pérez, 2013), a la vez que puede ayudar a tomar decisiones sobre el seguimiento de una problemática entre sus ejes emergentes y/o descendentes e identificar cambios significativos en la manera de estudiar esta variable.

El mapeo científico es una herramienta que toma un conjunto de registros bibliográficos de un campo de investigación y genera una descripción general del dominio del conocimiento subyacente que ayuda a entender puntos críticos que evaluan el impacto de los estudios, redes de colaboración y entidades cooperantes (Chen \& Song, 2019) como resultado de diversos indicadores bibliométricos y una evolución dinámica y estructural de términos específicos (Moreno-Guerrero et al., 2020). Esta metodología es beneficiosa por su aproximación a una mejor comprensión de la multidimensionalidad de las adicciones, la complejidad geográfica, la heterogeneidad de los resultados y evolución en el campo científico.

Si bien es cierto, esta heterogeneidad puede permitir una visión general para un seguimiento documental, también puede hacer invisible puntos de referencia por la magnitud de los grupos de investigación, fuentes de producción, revistas en proceso de indización y número de artículos publicados (Ávila-Toscano et al., 2014; Vera-Villarroel et al., 2010). Las revistas peruanas forman parte de los grupos emergentes, que pueden generar un valioso alcance, ya que su aproximación colabora con un mayor entendimiento de estudios regionales en las tendencias específicas de investigación (Morales, 2016; Santillán-Aldana et al., 2017)

La utilidad de este trabajo es importante dentro del campo de las adicciones psicológicas en el Perú y en Latinoamérica, por su extensión y posible desarrollo de políticas de investigación en este campo, ya que permitirá realizar un balance objetivo sobre el conocimiento publicado 
y duplicar esfuerzos innecesarios con resultados previsibles con un gasto de recursos materiales y humanos injustificado (Perestelo-Pérez, 2013).

La selección de las publicaciones se desarrolló mediante consultas en Scielo Citation Index (SCI) de Web of Science, en el Sistema Regional de Información en Línea para Revistas Científicas de América Latina, el Caribe, España y Portugal (Latindex) y en el buscador Google Scholar. Asimismo, también se ha recogido publicaciones que en el momento de las consultas realizadas, no estaban indizadas y en algunos casos al revisar las referencias de publicaciones que fueron conocidas por los investigadores.

La búsqueda incluyó revistas de diferentes áreas del conocimiento como: psicología, ciencias de la salud, educación, ciencias sociales, humanidades y algunas de carácter multidisciplinario. Asimismo, se consideraron estudios realizados en diversos tipos de adicción como internet, redes sociales, videojuegos, trabajo, etc. (Arias \& Masías, 2017; Calle-Sarmiento et al., 2013; Contreras, 2015; Delgado et al., 2016; Domínguez \& Ybañez, 2016; Espinoza, 2018; Flores et al., 2015; Gamero et al., 2016; Gómez-Burns \& Arela-Bobadilla, 2017; Martino, 2014; Mejía et al., 2014; Morales, 2015; Padilla-Romero \& Ortega-Blas, 2017; Salas, 2014; Sanchez-Meca \& Botella, 2010; Uchuypoma, 2017; Valencia, 2016; Vara, 2017) . Los objetivos del estudio fueron analizar el desarrollo de la investigación de las adicciones comportamentales en revistas peruanas en un período que va de 2003 a 2019; se analizaron características del medio de publicación, nivel de visibilidad y de indización, el área y la institución de pertenencia; analizar la producción investigativa tomando en cuenta aspectos metodológicos empleados (diseños, participantes e instrumentos) y el alcance de la citación de los estudios.

\section{Método}

\section{Tipo de estudio}

Se realizó un estudio documental y se utilizó mapeo científico e indicadores bibliométricos para el análisis de procesos relacionados con la cuantificación y evaluación de la producción científica (Moreno-Guerrero et al., 2020).

\section{Estrategia de búsqueda}

Para la búsqueda se tomaron en cuenta dos rutas específicas debido a la diversificación en la clasificación de las revistas científicas peruanas; en primer lugar, fueron identificadas todas las revistas peruanas que tuvieran como temática el componente de adicciones comportamentales que se hallaron en diversos repositorios, registros, cosechadoras y/o buscadores (e.g., Google Scholar), se tomó en cuenta el año 2003 como primer registro de esta variable hasta el 2019. Inicialmente se encontraron más de 120 documentos, al analizarlos con los criterios de inclusión y de exclusión planteados, se redujeron a 53, como datos de interés histórico, el primer artículo fue publicado el 2003 por la Revista de Psicología de la Pontificia Universidad Católica del Perú (PUCP), el año en el que se publicaron más artículos fue el 2016, y en la Universidad Peruana Cayetano Heredia se encontraron hasta cinco revistas que publicaron artículos relacionados con el tema de la presente investigación.

Para el siguiente paso, fueron identificadas las revistas que pertenecían a la fecha a una base de datos como mínimo criterio (indizadas en Scielo Citation Index).En general, la estrategia de búsqueda abarcó los conceptos relevantes en las conductas adictivas sin sustancias (Topic $=$ TS) para familia de palabras de adicciones comportamentales. Esta estrategia fue formulada para los criterios más relevantes en conductas adictivas basadas en la literatura actual y la identificación de variantes en las palabras de búsqueda. En este proceso fueron identificados 72 documentos en los que se aplicaron los mismos criterios de inclusión y exclusión para finalmente seleccionar 11 artículos para el análisis. Los datos de las revistas indizadas fueron extraídos de Web of Science (Scielo Citation Index).

\section{Unidad de análisis: criterios de inclusión y selección de artículos}

Se consideró algunos criterios de inclusión y de exclusión de los artículos inicialmente seleccionados. Entre los criterios de inclusión se propuso: que hayan sido publicados en alguna revista científica peruana, indizada o no hasta marzo de 2019, en sus diferentes tipos documentales (teórico, 
empírico, metodológico, etcétera) publicados en revistas en plataformas física o digital. Asimismo, se consideraron algunos criterios de exclusión como: artículos que trataban del tema de internet o de redes sociales desde perspectivas diferentes a las adicciones o uso problemático, estudios con una fuente ambigua de publicación que no indicaban la revista de pertenencia (literatura gris) $u$ otros tipos documentales de baja citación como cartas al editor, editoriales y capítulos de libro.

\section{Procedimiento}

En la primera fase del estudio se localizaron revistas peruanas según su presentación (virtuales e impresas). Una vez identificadas las revistas, fue establecida la primera fecha de publicación sobre el tema de conductas adictivas sin sustancias para determinar el rango de búsqueda (2003 - 2019) que incluyó de manera general bases de datos, evaluadoras y cosechadoras como SCI, Redalyc, Latindex y Google Scholar. Se utilizaron algunas palabras clave como "social network addiction" OR "social network dependence" OR "facebook addiction" OR "facebook dependence" $O R$ "Problematic social network use" OR "internet addiction" $O R$ "smartphone problematic use" $O R$ "smartphone addiction" $O R$ "instagram addiction" $O R$ "problematic instagram use" $O R$ workaholism OR "work addiction" OR addiction*
$O R$ "dependence of videogame" $O R$ "videogame addiction" $O R$ "dependence" $O R$ "dependencia" $O R$ "adicciones" $O R$ "conducta adictiva". Se incluyeron a una base de datos construida para los propósitos del estudio donde se analizaron las frecuencia de las publicaciones de las revistas, características metodológicas y fuentes instrumentales de los artículos.

\section{Análisis de datos}

Fue extraída una base de datos bibliográfica de SCI, con el fin de realizar un análisis de redes de información con las palabras clave de autores, co-autoría y organizaciones normalizadas por modularidad (linlog) calibrando la atracción de términos < 3 (Waltman \& Van Eck, 2013), además fueron tomados en cuenta conteos de productividad, índice $h$ y otros índices que no están influenciados por la temporalidad como el índice $m$ y el índice $g$ que toma en consideración la existencia de artículos citados que no entran dentro del índice $h$.

\section{Resultados}

Los análisis buscan describir el desarrollo de las publicaciones en el área de las adicciones comportamentales, hechas desde 2003 hasta marzo de 2019.

Figura 1

Conteo de las publicaciones en adicciones comportamentales

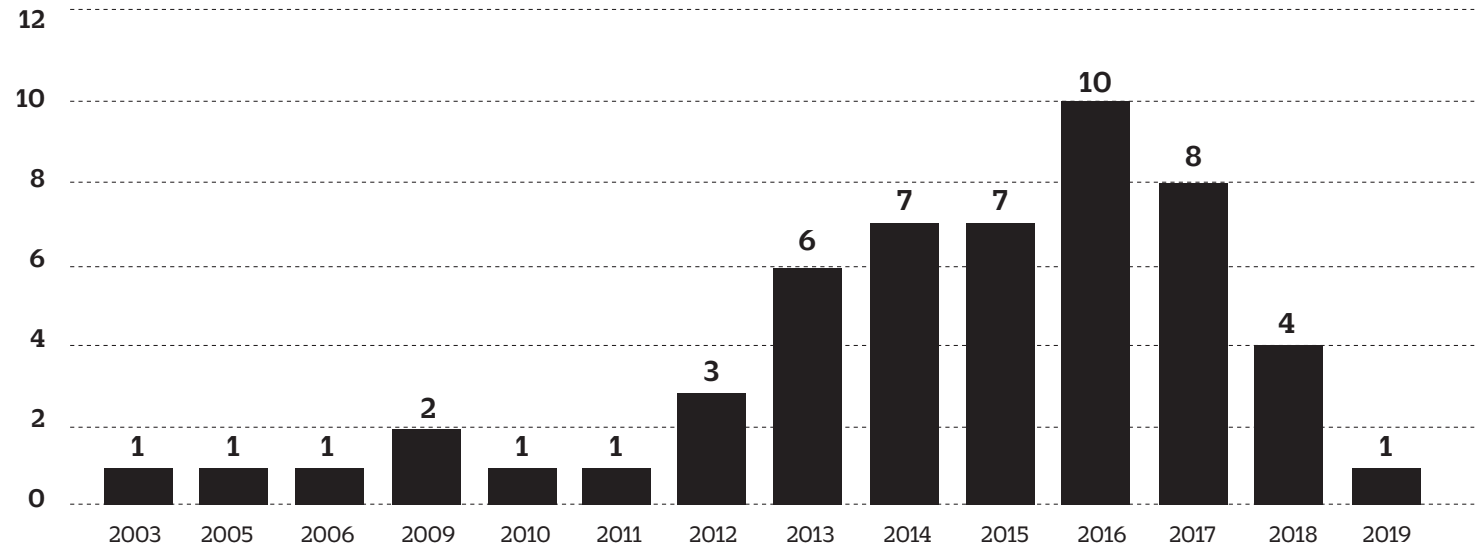




\section{Características de las publicaciones}

Entre los años 2003, 2005 y 2006 se publicó apenas un artículo por año, en 2004, 2007 y 2008 no se encontraron trabajos publicados. Solo a partir de 2012 se nota un incremento de publicaciones y es en 2016 el año en el que se alcanza la mayor producción con 10 artículos (véase figura 1).

\section{Frecuencia de autoría}

$\mathrm{Al}$ analizar la cantidad de autores que firman los artículos se evidencia que el $38 \%$ de artículos tiene autoria única , el 25\% de artículos fue publicado por dos autores, con tres autores se publicaron el
$15 \%$ de artículos y con cuatro autores se encontraron tres artículos (6\%). En el otro extremo con cantidades mayores, se encontraron que dos artículos fueron firmados por 10 autores cada uno, además aparece un artículo por cada grupo con 12,13 y 18 autores solo reportaban entre un $4 \%$ a $2 \%$.

\section{Análisis de estudios por revista e institución}

La revista que posee la mayor cantidad de artículos publicados sobre adicciones comportamentales fue Hamut'ay, que publicó cinco artículos y pertenece a la Universidad Alas Peruanas. Cinco

\section{Tabla 1}

Descripción de revistas por pertenencia, disciplina y acumulado de estudios

\begin{tabular}{|c|c|c|c|c|}
\hline Revista & Institución & Area/Discip. & Registro & \# art \\
\hline Rev. Psicológica Herediana & $\mathrm{UPCH}$ & Psicología & Latindex & 1 \\
\hline Rev. de Enfermería Herediana & $\mathrm{UPCH}$ & CC. Salud & Latindex & 1 \\
\hline Acta Psicológica Peruana & U. Autónoma & Psicología & ninguna & 3 \\
\hline Avances en Psicología & UNIFE & Psicología & Latindex & 3 \\
\hline Rev. de Invest. En Psicología & UNMSM & Psicología & Latindex & 2 \\
\hline Cultura & Asoc. Doc. Univ. S. Martín Porr (USMP) & Multitemát. & Latindex & 2 \\
\hline Educationis Momentum & Univ. Cat. San Pablo (UCSP-Arequipa) & Educaqión & ninguna & 1 \\
\hline Rev. De Psicología (Arequipa) & UCSP-Arequipa & Psicología & Latindex & 2 \\
\hline Hamut'ay & Univ. Alas Peruanas & CC. Salud & Latindex & 5 \\
\hline Horizontes de la Ciencia & Univ. Nac. Del Centro del Perú & Educación & Latindex & 2 \\
\hline Persona & Univ. De Lima & Psicología & Latindex & 3 \\
\hline Rev. Científica de CC. De la Salud & Univ. Peruana Unión & CC. Salud & Latindex & 3 \\
\hline Rev. De Psicología de Arequipa & Colegio de Psicólogos de Arequipa & Psicología & Latindex & 1 \\
\hline Rev. de Psicología & $U C V$ & Psicología & Latindex & 1 \\
\hline Rev. de Inv. y Casos en Salud-CASUS & UCSS & CC. Salud & Latindex & 2 \\
\hline Rev. Digital EOS Perú & Instituto Psicopedagógico EOS Perú & Educación & Latindex & 1 \\
\hline Rev. PAIAN & Univ. Señor de Sipán & Psicología & ninguna & 1 \\
\hline Rev. Peruana de Epiemiología & Sociedad Per. De Epidemiología & CC. Salud & Latindex & 1 \\
\hline Rev. De la Soc. Per. De Med. Interna & Soc. Peruana de Medicina Interna & CC. Salud & Latindex & 1 \\
\hline Rev. Per.de Psic. Y Trab. Social & UIGV & Psicología & Latindex & 2 \\
\hline Total de artículos & & & & 38 \\
\hline
\end{tabular}

Nota. art = número de artículo 
revistas publicaron tres artículos cada una: Avances en Psicología, Persona, Revista Científica de Ciencias de la Salud, Revista de Psicología de la PUCP y Acta Psicológica Peruana. Nueve revistas publicaron dos artículos y 15 han publicado un solo artículo. Al considerar la disciplina o el área al que pertenecen las revistas se encuentra que la mayor cantidad de artículos (24) y de revistas (13) son de psicología, seguida muy de cerca por publicaciones hechas en el área de las ciencias de la salud, que son revistas de diferentes especialidades médicas y de enfermería (22 artículos y 12 revistas), en el área de la educación se encontraron cinco artículos y cuatro revistas; y, una revista multitemática, que publicó dos artículos.

Además las revistas que publicaron artículos sobre adicciones comportamentales se encontraron un total de 22 instituciones, 16 de ellas universitarias; cinco pertenecen a institutos o sociedades médicas especializadas y una revista pertenece a una Asociación de Docentes universitarios. En la Universidad Peruana Cayetano Heredia se encontraron cinco revistas, una de psicología y las restantes de diferentes áreas médicas y de enfermería, en la Universidad de San Martín de Porres se encontraron dos revistas de facultades y una de la Asociación de Docentes; en la Universidad Nacional Mayor de San Marcos (UNMSM) y en la Universidad Católica San Pablo de Arequipa se encontraron dos revistas en cada una, y, en las demás se halló una revista (ver Tabla 1). Los 53 artículos seleccionados fueron publicados en 38 revistas, al revisar su mayor logro de indización alcanzado por las revistas se encontró que cuatro no han alcanzado aún indización alguna, 16 de ellas están consideradas en el catálogo de Latindex.

\section{Características metodológicas de los artículos}

Dentro de las características metodológicas evaluadas se evidenció que hasta el momento no existen estudios cualitativos, 43 de ellos usan datos cuantitativos (81\%) primarios o secundarios y 10 (19\%) son de tipo teórico narrativo (Hernández et al., 2014), Entre los 43 estudios cuantitativos se halló que 16 utilizaron muestras de adolescentes (34.78\%), 23 estudios trabajaron con participantes jóvenes (53.5\%), dos trabajaron con población adulta, uno utilizó análisis de datos censales y uno utilizó historias clínicas de pacientes.

Se configuraron tres grandes clases de artículos según el tipo de diseño: cuatro artículos (7.5\%) de tipo instrumental, referidos a la construcción o validación de instrumentos; 10 de tipo teórico narrativo (19\%) y dos (3.7\%) contenían análisis secundarios de datos de una encuesta nacional previamente desarrollada con propósitos diferentes y de historias clínicas de pacientes internados en un hospital psiquiátrico); y, 37 fueron estudios empírico cuantitativos (69.9\%), de tipo descriptivo o asociativo (Ato et al., 2013). Además, teniendo en cuenta la técnica utilizada para la recolección de datos, se encontró que 12 de ellos (22.2\%), utilizaron la revisión de tipo bibliográfico, 10 de ellos fueron estudios narrativos y dos usaron análisis secundario. Los 41 restantes (77.8\%) utilizaron la técnica de la encuesta, generalmente cuestionarios de tipo autoinforme.

En cuanto a los instrumentos que se han utilizado en los 41 estudios empíricos, se encontró que se utilizaron 15 escalas o cuestionarios y ocho encuestas ad hoc construidas para el estudio desarrollado en específico. De los 15 instrumentos, 10 de ellos (66.6\%) fueron construidos y/o validados en el Perú, cinco de ellos no fueron validados (33.3\%). Los instrumentos más usados fueron dos: 10 estudios (23.6\%) emplearon el Cuestionario de Adicción a Redes Sociales (ARS, Escurra \& Salas-Blas, 2014), nueve (21.4\%) emplearon el Internet Addiction Test (IAT, Young, 1998).

Otro tema de análisis que se planteó, fue revisar el diseño metodológico utilizado. De acuerdo con este criterio la mayor cantidad de estudios hallados y que fueron materia de análisis correspondiendo al tipo predictivo (Ato et al., 2013) o correlacionales (Hernández \& Mendoza, 2018), 22 estudios en total (41.5\%), luego siguieron los estudios descriptivos y los estudios de revisión teórica. Dentro de los estudios con diseño correlacional (41.5\%), se encontró que los temas que se han correlacionado con alguna de las adicciones comportamentales fueron los siguientes: conflicto trabajo-familia, relaciones intrafamiliares $\mathrm{y}$ funcionamiento familiar (5 estudios $=22.7 \%$ ), personalidad (3 estudios $=13.6 \%$ ), agresividad (3 estudios $=13.6 \%$, habilidades sociales ( 3 estudios $=$ 
13.6\%); procrastinación ( 2 estudios = 9\%); la depresión /soledad con dos estudios ( 2 estudios $=9 \%$ ); $\mathrm{y}$, con un estudio ( $4.55 \%$, cada uno) autoestima, hábitos de estudio, impulsividad y desempeño académico.

\section{Objetos de estudio desarrollados}

Otro aspecto del análisis que se propuso para este trabajo tiene que ver con el objeto específico estudiado, se encontró que la adicción a internet es el objeto de estudio más frecuente (39.6\%); en segundo término, las publicaciones sobre la adicción a las redes sociales (25.4\%), luego se encontraron las adicciones a videojuegos con un $9.4 \%$ de publicaciones, al igual que las adicciones tecnológicas. Hay que notar que no se han encontrado evidencias sobre otras adicciones como a las compras, al ejercicio, al sexo, al afecto, etc. (<4\%), que son materia de publicaciones en otros lugares del mundo.

Las adicciones estudiadas han sido analizadas con otras variables relacionadas con la personalidad, Arias y Masías (2016) encontraron que no existe relación entre la cantidad de tiempo dedicado al uso de la computadora y las medidas de las dimensiones de la personalidad desde la perspectiva de Eysenck; Torres (2015) relacionó el consumo de internet y la personalidad, encontrando que el neuroticismo se relaciona positivamente con el consumo de internet en varones de 18 a 25 años.

Astonitas (2005) usó el NEO-PI-R y una encuesta que ella elaboró sobre riesgo de adicción a internet, encontró que el neuroticismo se correlaciona positivamente con la cantidad de síntomas de adicción a internet; encontró también que las facetas sentido del deber, aspiraciones de logro, autodisciplina y reflexión, de la dimensión conciencia correlacionan negativamente con la cantidad de síntomas de adicción a internet, cuestión que se corrobora con el estudio de Challco et al. (2016), quienes hallaron correlaciones negativas entre diversas dimensiones del Cuestionario de autocontrol infantil y adolescente con la adicción a redes sociales. En esta misma línea, se puede incluir el trabajo desarrollado por Clemente et al. (2018) que correlaciona positivamente adicción a redes sociales e impulsividad; $y$, la obsesión por las redes sociales se correlaciona positivamente con premeditación, mientras que inversamente con urgencia negativa.

\section{Mejora de la calidad: evolución de las es- tudios en adicciones comportamentales en revistas indizadas}

El proceso de indización ayuda a incorporar mejoras en los procesos editoriales, el tratamiento y filtrado de los manuscritos, para posteriormente ser incluidas en las bases de datos e índices internaciones (Chavarro et al., 2018; Navas-Fernández et al., 2018; Rousseau, 2002). Además, las revistas que se encuentran indizadas tienen más visibilidad y accesibilidad de las publicaciones a diferencia de las revistas que se encuentran en otras plataformas (e.g., registros, repositorios, cosechadoras o buscadores).

En la figura 2 se muestra el grafo para la red de palabras clave. Se utilizó palabras clave (autores) para construir las redes de co-ocurrencia. Esto implica la frecuencia de aparición de las palabras clave como una ruta de las tendencias de investigación en las adicciones comportamentales. Las palabras con una mayor frecuencia son representadas por el tamaño del nodo ( > 2 umbrales) para el intervalo de tiempo (2006 - 2019) que configuran cuatro clusters como diseño, problemas conducta disruptiva, factor de riesgo y uso de instrumentos.

Para el grafo de redes de co-autoría por documento (véase figura 3), el tamaño del nodo muestra una mayor colaboración con respecto a los demás documentos ( $>3$ documentos) para el intervalo de tiempo (2006 - 2019) que configuran seis clusters que corresponden al número de colaboración, el cluster con mayor actividad (rojo) corresponde a una red de coautoría a través del tiempo.

Las redes de colaboración institucional (véase figura 4) muestran que la UNMSM, para el intervalo 2006-2019 tiene una mayor colaboración con respecto a las demás instituciones. En los nodos periféricos no conectados al grafo, la Universidad Veracruzana (UV) es la única institución internacional que figura dentro de la frecuencia de instituciones en adicciones comportamentales en el Perú (con una sola institución). Además, la Diresa-Callao y la UCH configuran una pequeña colaboración de manera conectada pero periférica con el grafo. 
Figura 2

Co-ocurrencia de palabras clave en adicciones comportamentales

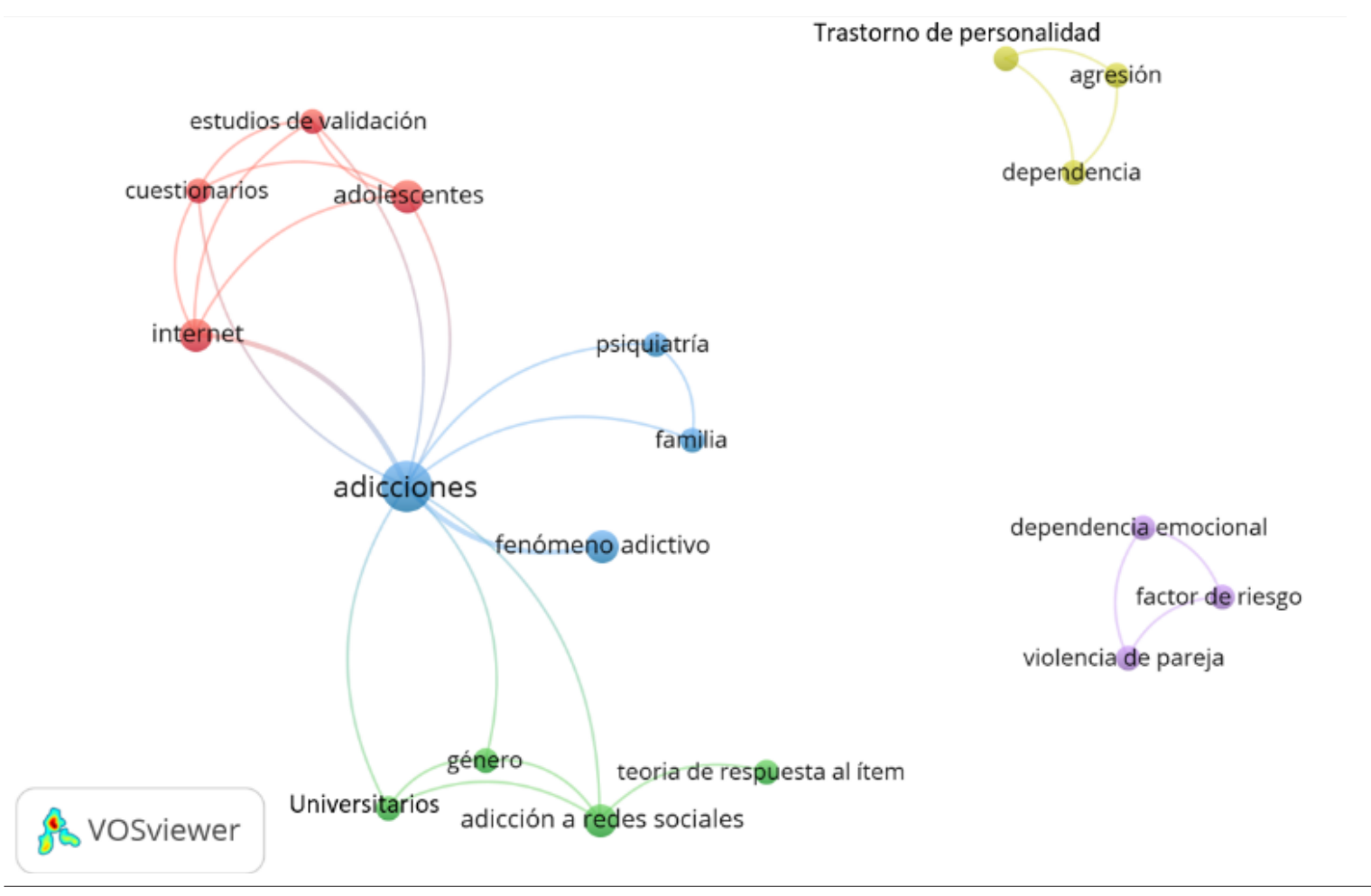

Figura 3

Redes de coautoría en adicciones comportamentales
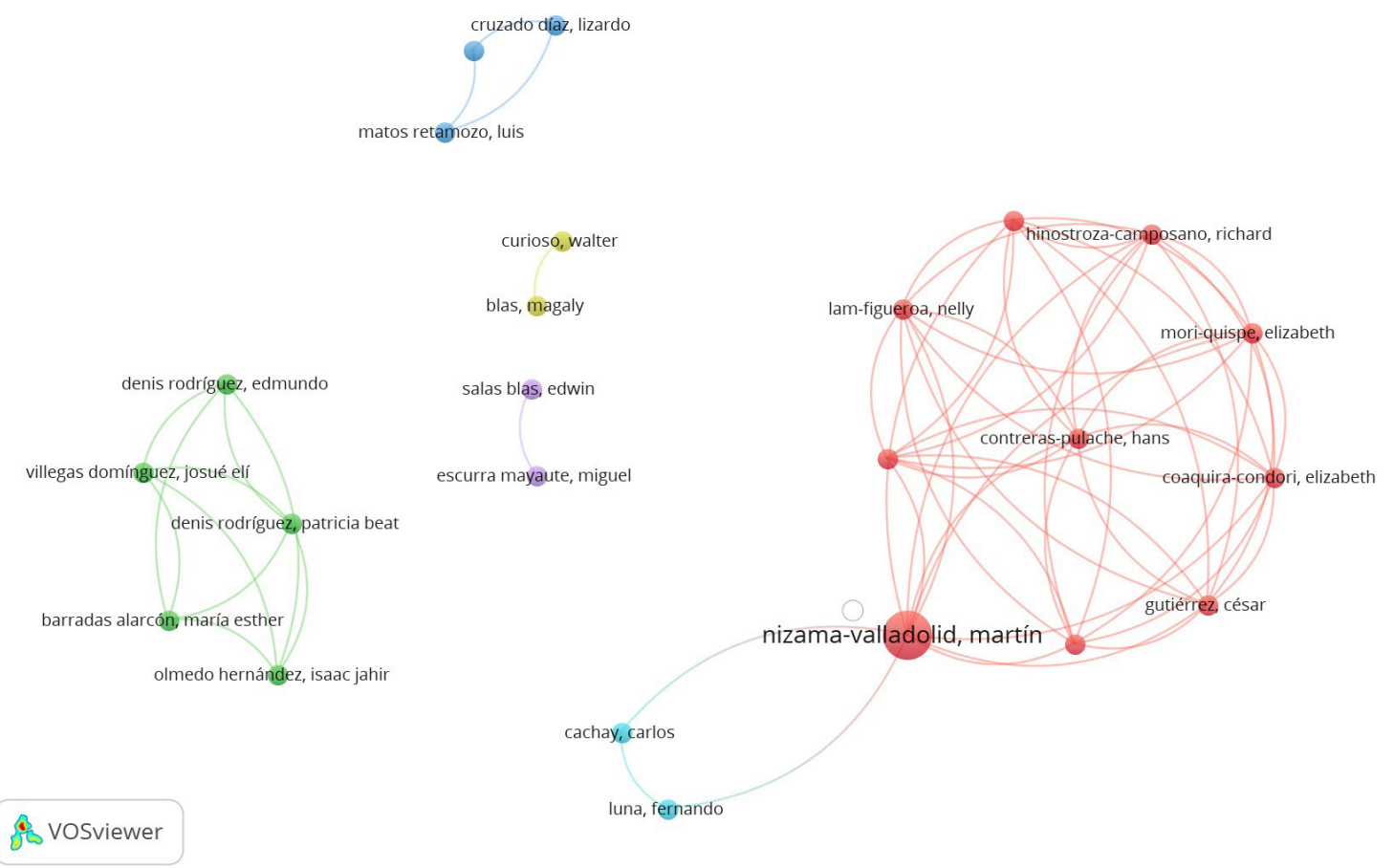
Figura4

Redes de colaboración institucional por autor corresponsal

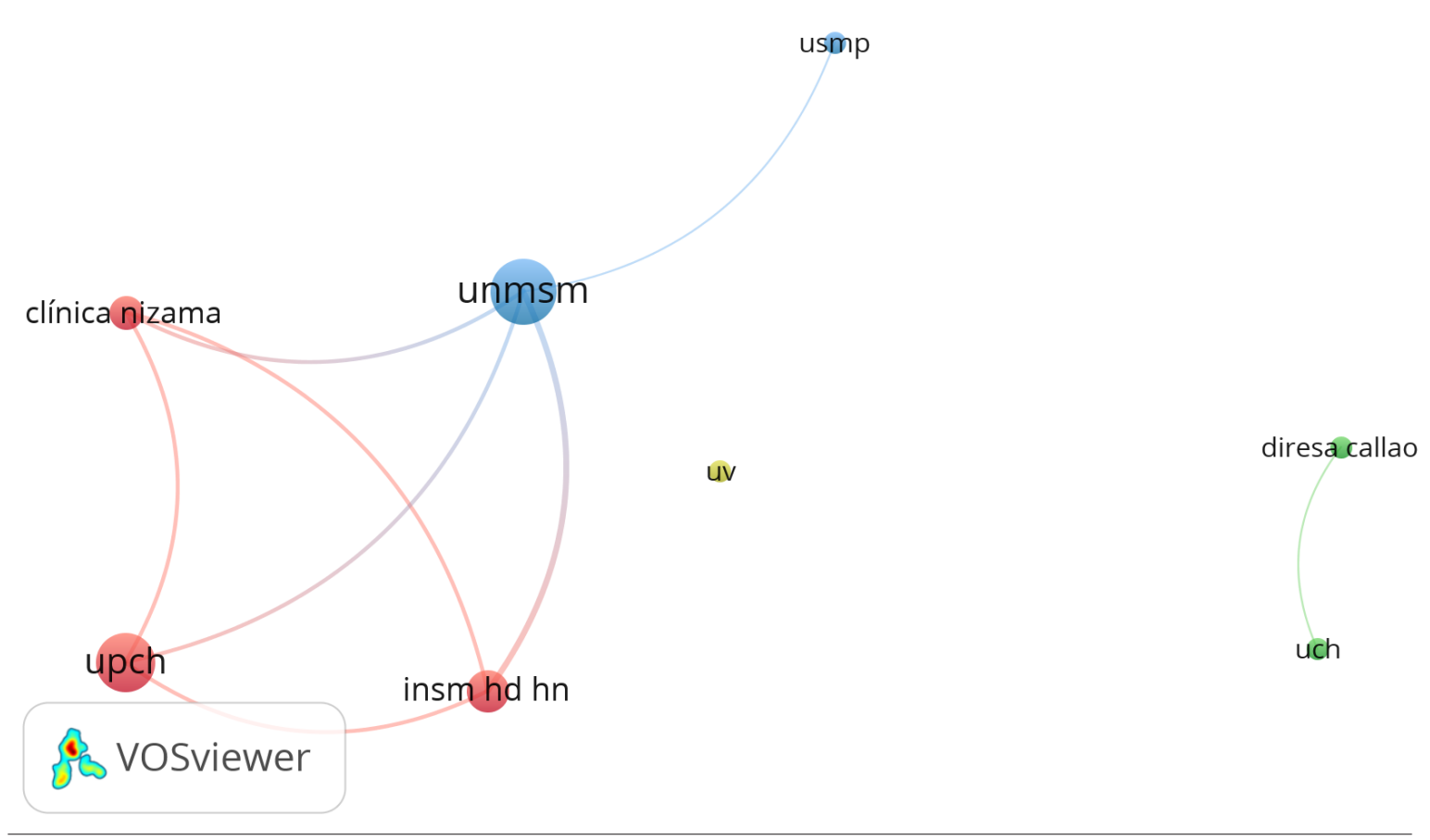

Nota. unmsm = Universidad Nacional Mayor de San Marcos; upch = Universidad Peruana Cayetano Heredia; insm hd hn = Instituto Nacional de Salud Mental "Honorio Delgado - Hideyo Noguchi"; uch = Universidad de Ciencias y Humanidades; $u v=$ Universidad de Valencia

El acumulado de citas (TC) de los estudios reportan entre dos a seis citaciones generales, en el caso del artículo de Escurra y Salas-Blas (2014) es el que reporta más citaciones para esta base de datos $(\mathrm{TCa}=.43$ ). Además fue extraído el índice $h$ del primer autor de los estudios que no evidenció influencia por la capacidad de citación en adicciones comportamenles, solo un estudio reportó financiamiento independientemente de la recogida de datos (ver tabla 2).

Las revistas peruanas, a partir del 2006, han presentado por lo menos un valor de índice $h=1$, en SCI, con un ligero incremento acumulado de publicaciones ( $g=2$ ) como es el caso de la Revista Herediana, sin embargo, la mayor influencia temporal hasta el momento lo evidencia la revista Liberabit $(m=.14)$. El grupo restante de revistas, a pesar de tener un acumulado heterogéneo (e.g., NP $=2$ ), no cuenta con valores para los indicadores (tabla 3). Además, en el contexto peruano no se han identificado citas locales entre los autores, sin embargo solo tres artículos muestran citas globales (CG) como Cruzado et al., 2006 (CG = 6) Escurra \& Salas-Blas, 2014 (CG = 3) y Lam-Figueroa et al., 2011 ( $C G=2)$.

\section{Discusión}

Los investigadores peruanos conocen bien las carencias que el país tiene en cuanto a revistas científicas (Salas-Blas, 2019). Hay varios asuntos que este estudio pone en la superficie: primero, no existe la cantidad suficiente de revistas para satisfacer la producción actual; segundo, las revistas que actualmente existen en el medio todavía no tienen certificaciones y aparentemente un tenue impacto para competir con otras contextos mejor posicionados, lo que trae como consecuencia que la mayor parte de investigadores tenga la necesidad de mirar al exterior, con el fin de adecuarse a 
Tabla 2

Artículos de adicciones comportamentales en revistas indizadas en SCI

\begin{tabular}{|c|c|c|c|c|c|c|c|}
\hline Autor (es) & Año & Título & Revista & $\begin{array}{l}\text { Financia- } \\
\text { miento }\end{array}$ & TC & TCa & $\begin{array}{l}\text { h-index } \\
\text { (a)ç }\end{array}$ \\
\hline $\begin{array}{l}\text { Nizama-Valladolid, Luna y } \\
\text { Cachay }\end{array}$ & 2019 & $\begin{array}{l}\text { La adiccionología una } \\
\text { especialidad médica y } \\
\text { subespecialidad de la } \\
\text { psiquiatría }\end{array}$ & $\begin{array}{l}\text { Revista de } \\
\text { la Facultad } \\
\text { de Medicina } \\
\text { Humana }\end{array}$ & no & 0 & 0 & 1 \\
\hline $\begin{array}{l}\text { Olmedo Hernández, Denis } \\
\text { Rodríguez, Barradas Alarcón, } \\
\text { Villegas Domínguez y Denis } \\
\text { Rodríguez }\end{array}$ & 2019 & $\begin{array}{l}\text { Agresividad y conducta } \\
\text { antisocial en individuos } \\
\text { con dependencia al } \\
\text { teléfono móvil: un posible } \\
\text { factor criminogénico }\end{array}$ & $\begin{array}{l}\text { Horizonte } \\
\text { Médico (Lima) }\end{array}$ & si & 0 & 0 & 0 \\
\hline Araujo-Robles & $2016 \mathrm{~b}$ & $\begin{array}{l}\text { Indicadores de adicción } \\
\text { a las redes sociales en } \\
\text { universitarios de Lima }\end{array}$ & $\begin{array}{l}\text { Revista } \\
\text { Digital de } \\
\text { Investigacin } \\
\text { en Docencia } \\
\text { Universitaria }\end{array}$ & no & 0 & 0 & 1 \\
\hline Morales Quispe & 2015 & $\begin{array}{l}\text { Uso de las tecnologías } \\
\text { de información y } \\
\text { comunicación en } \\
\text { adolescentes de las } \\
\text { instituciones educativas } \\
\text { públicas del Distrito Mi } \\
\text { Perú }\end{array}$ & $\begin{array}{l}\text { Anales de la } \\
\text { Facultad de } \\
\text { Medicina }\end{array}$ & no & 0 & 0 & 0 \\
\hline Nizama-Valladolid & 2015 & $\begin{array}{l}\text { Innovación conceptual en } \\
\text { adicciones: Segunda parte }\end{array}$ & $\begin{array}{l}\text { Revista } \\
\text { de Neuro- } \\
\text { Psiquiatra }\end{array}$ & no & 0 & 0 & 1 \\
\hline Nizama-Valladolid & 2015 & $\begin{array}{l}\text { Innovación conceptual en } \\
\text { adicciones: Primera parte }\end{array}$ & $\begin{array}{l}\text { Revista } \\
\text { de Neuro- } \\
\text { Psiquiatra }\end{array}$ & no & 0 & 0 & 1 \\
\hline Escurra y Salas-Blas & 2014 & $\begin{array}{l}\text { Construcción y validación } \\
\text { del cuestionario de } \\
\text { adicción a redes sociales } \\
\text { (ARS) }\end{array}$ & Liberabit & no & 3 & 0.43 & 1 \\
\hline $\begin{array}{l}\text { Lam-Figueroa, Contreras- } \\
\text { Pulache, Mori-Quispe, } \\
\text { Nizama-Valladolid, } \\
\text { Gutiérrez, Hinostroza- } \\
\text { Camposano, Torrejón Reyes, } \\
\text { Hinostroza-Camposano, } \\
\text { Coaquira-Condori y } \\
\text { Hinostroza-Camposano }\end{array}$ & 2011 & $\begin{array}{l}\text { Adicción a internet: } \\
\text { desarrollo y validación } \\
\text { de un instrumento en } \\
\text { escolares adolescentes de } \\
\text { Lima, Perú }\end{array}$ & $\begin{array}{l}\text { Revista } \\
\text { Peruana de } \\
\text { Medicina } \\
\text { Experimental } \\
\text { y Salud } \\
\text { Publica }\end{array}$ & no & 2 & 0.20 & 2 \\
\hline Curioso y Blas & 2007 & $\begin{array}{l}\text { Internet, adicción, } \\
\text { cibersexo e infecciones de } \\
\text { transmisión sexual en el } \\
\text { Perú: ¿Ficción o realidad?. }\end{array}$ & $\begin{array}{l}\text { Revista } \\
\text { Medica } \\
\text { Herediana }\end{array}$ & no & 0 & 0 & 4 \\
\hline $\begin{array}{l}\text { Cruzado Díaz, Matos } \\
\text { Retamozo y Kendall Folmer }\end{array}$ & 2006 & $\begin{array}{l}\text { Adicción a internet: Perfil } \\
\text { clínico y epidemiológico } \\
\text { de pacientes } \\
\text { hospitalizados en un } \\
\text { instituto nacional de salud } \\
\text { mental }\end{array}$ & $\begin{array}{l}\text { Revista } \\
\text { Medica } \\
\text { Herediana }\end{array}$ & no & 6 & 0.40 & 1 \\
\hline
\end{tabular}

Nota $. T C=$ total de citas; $T C a=$ total de citas por año; (a) índice $h$ primer autor 
Tabla 3

Revistas que publicaron estudios sobre adicciones comportamentales en SCI

\begin{tabular}{|c|c|c|c|c|c|c|}
\hline Revista & PA & $\mathbf{h}$ & g & $\mathbf{m}$ & TC & $\# \mathbf{p}$ \\
\hline Revista Medica Herediana & 2006 & 1 & 2 & .07 & 6 & 2 \\
\hline Liberabit & 2014 & 1 & 1 & .14 & 3 & 1 \\
\hline Revista Peruana de Medicina Experimental y Salud Publica & 2011 & 1 & 1 & .10 & 2 & 1 \\
\hline Revista de Neuro-Psiquiatra & 2015 & 0 & 0 & 0 & 0 & 2 \\
\hline Anales de la Facultad de Medicina & 2015 & 0 & 0 & 0 & 0 & 1 \\
\hline Horizonte Médico (Lima) & 2019 & 0 & 0 & 0 & 0 & 1 \\
\hline Revista de la Facultad de Medicina Humana & 2019 & 0 & 0 & 0 & 0 & 1 \\
\hline Revista Digital de Investigación en Docencia Universitaria & 2016 & 0 & 0 & 0 & 0 & 1 \\
\hline
\end{tabular}

Nota. $T C=$ total de citas $;=$ índice $h ; g=$ índice $g ; m=$ índice $m ; \# p=N^{\circ}$ de publicaciones

los sistemas de calificación por organismos reguladores de la universidad peruana (Superintendencia Nacional de Educación Superior Universitaria - Sunedu) y del Consejo Nacional de Ciencia, Tecnología e Innovación (Concytec).

En cuanto a la visibilidad las revistas peruanas en esta área en su mayoría no cuentan característica ni con la internacionalización, que son deseables para atraer publicaciones de académicos de primer nivel; los investigadores reconocidos buscan publicaciones indizadas en bases como Scopus o Web of Science (WOS) que en el Perú aún son muy pocas.

Nuestra propuesta revisa la evidente situación de crisis ocasionada por el poco desarrollo de las revistas científicas, cuyo origen podría tener como una de sus causas, una inadecuada gestión de las mismas, como se sostiene en Salas-Blas (2019). Para el propósito de esta investigación se encontraron solo 30 revistas (de varias áreas), de ellas cuatro aún no se encuentran en ningún catálogo o base de datos. En ese sentido, en el caso de psicología, existen más de 40 centros de formación de psicólogos y solamente 16 de ellas tienen una revista científica, tres han alcanzado indizarse en Scielo y solo una en Scopus y WOS.

La producción de Colombia, Argentina, México o Brasil supera ampliamente este número de revistas peruanas en esta temática; más si se tiene en cuenta que en los tiempos actuales se exige al mundo académico producción científica y evidentemente esto no se puede dar si no existen publicaciones científicas que faciliten la producción de artículos científicos. En 17 años que involucra la búsqueda que se ha realizado para este estudio (2003 - 2019) sólo se encontraron 53 artículos que fueron materia de análisis, un promedio de 3.1 artículos por año.

En cuanto a las características metodológicas de los artículos analizados, alrededor del $80 \%$ de los artículos contienen datos cuantitativos, casi el $20 \%$ son artículos de revisión teórica. Más de la mitad estudiaron muestras de jóvenes, seguido por estudios hechos con adolescentes, no se encontraron estudios con niños, ni con padres de familia o profesores, contextos que son importantes como elementos para analizar el comportamiento adictivo o dependiente. Una de las cuestiones más llamativas es que solo cuatro estudios son de tipo instrumental, dos de ellos tratan de la construcción de instrumentos (Lam-Figueroa et al., 2011; Escurra \& Salas-Blas, 2014) y los otros dos validaron instrumentos ya existentes. Existen numerosos estudios que emplean instrumentos no validados en el medio, hecho que resta confiabilidad a los datos que hallaron y comunicaron.

Es de resaltar que uno de los problemas más importantes que se tiene en el Perú para desarrollar investigación en condiciones adecuadas es la carencia de instrumentos validados. Se en- 
cuentra que el cuestionario ARS de Escurra y Salas-Blas (2014) es el más empleado por los investigadores de las adicciones a redes sociales, seguido por el IAT de Young, validado por Matalinares et al. (2014), por lo que el IAT q a pesar de su antigüedad, sigue siendo empleado no solamente en el Perú. Asimismo, el objeto de estudio más frecuente que se ha investigado es la adicción a internet, seguido de la adicción a redes sociales y de la adicción a videojuegos, no se encontraron estudios relacionados con temas de adicciones a las compras, al sexo, al ejercicio, la comida, o, a los afectos. Una posible causa de la falta de investigación en las áreas señaladas, podría ser la falta de instrumentos, ligado al poco conocimiento que se tiene del tema de las adicciones psicológicas. Finalmente, en cuanto al diseño metodológico, la mayor parte de los estudios cuantitativos es de tipo predictivo o correlacional (Ato et al., 2013; Hernández \& Mendoza, 2018), siguen los estudios descriptivos y los de revisión teórica.

No se encontraron estudios cualitativos, ni de tipo experimental que tengan que ver con experiencias de tratamiento o de prevención en conductas adictivas. Esto pone en relieve el nivel en el que se encuentra la investigación de las adicciones comportamentales en el Perú. A diferencia de otros países en los que este campo de investigación produce más evidencias que incluyen la evaluación de riesgos, tratamiento y prevención independientemente del tipo de comportamiento adictivo.

Sin embargo, este trabajo no está exento de limitaciones, como es el rango de estudio en comportamientos adictivos en revistas peruanas y su leve crecimiento, por lo que tener resultados documentales completos es poco escalable. Además, la mayoría de los estudios son trasversales, lo que no permitiría hacer una línea de seguimiento extendible a la producción que complemente otros tipos documentales. Sumado a ello, un limitado número de revistas están indizadas en bases de datos, esto complicaría la reproducibilidad en la búsqueda de términos, debido a que no todas las revistas cumplen con este criterio o en buena medida forman parte de la literatura gris.

A pesar de ello, un punto importante fue que las revistas indizadas en SCI evidenciaron que las áreas relacionadas con salud han iniciado un incremento en su capacidad de citación, a diferencia de las revistas que no se encuentran indizadas, esto también puede ser influenciado por la falta de normalización del documento cosechado, que incluso no puede ser utilizable con certeza en una búsqueda sistematizada.

Entre algunas de las recomendaciones para mejorar la investigación en la línea de las adicciones comportamentales, se podría considerar la necesidad de realizar estudios instrumentales para adaptar o validar instrumentos ya existentes y que están en otros idiomas o requieren de una adecuación al contexto peruano; investigar más puntualmente los factores relacionados con la personalidad, el desarrollo, las motivaciones y el contexto en el que viven y se desarrollan los diferentes grupos que tienen problemas de adicciones comportamentales, esto permitiría desarrollar también programas de tipo preventivo; asimismo centrar la atención en los riesgos y las consecuencias que generan los comportamientos excesivos e incontrolados y las adicciones en diferentes grupos que por su naturaleza son muy sensibles (niños, adolescentes, mujeres, mujeres de tercera edad, etc.).

\section{Referencias}

Andreassen, C. S. (2015). Online social network site addiction: A comprehensive review. Current Addiction Reports, 2, 175-184. https://doi.org/10.1007/s40429015-0056-9

Apud, I. \& Romaní, O. (2016). La encrucijada de la adicción. Distintos modelos en el estudio de la drogodependencia. Health and addictions, 16(2), 115-125. http:// www.redalyc.org/articulo.oa?id=83946520005

Araujo-Robles, E. D. (2015). De la adicción a la adicción a internet. Revista Psicología Herediana, 10(1-2), 47-56. https://revistas.upch.edu.pe/index.php/RPH/article/view/3023

Araujo-Robles, E. D. (2016a). Indicadores de adicción a las redes sociales: un estudio en escolares de secundaria de Lima. Revista de Psicología, 18(2). http://revistas. ucv.edu.pe/index.php/revpsi/article/view/372

Araujo-Robles, E. D. (2016b). Indicadores de adicción a las redes sociales en universitarios de Lima. Revista Digital de Investigación en Docencia Universitaria, 10(2), 48-58. https://revistas.upc.edu.pe/index.php/ 
docencia/article/view/494

Arcaya, M., Martina, M., Gutiérrez, C., \& Romero, Y. (2012). Práctica de juegos de azar y su relación con la integración familiar en la población peruana. Revista Peruana de Epidemiología, 16(1), 1-7. http://sisbib. unmsm.edu.pe/BVRevistas/epidemiologia/v16_n1/ pdf/a06v16n1.pdf

Arias, W., \& Masías, M. A. (2016). Personalidad y uso de la Computadora en estudiantes de ingeniería de sistemas. Avances en psicología, 24(2), 159-166. http:// revistas.unife.edu.pe/index.php/avancesenpsicologia/article/view/151

Arias, W., \& Masías, M. A. (2017). Adicción a internet en cibernautas de cuatro distritos de Arequipa Metropolitana. Revista de Psicología de Arequipa, 7(1), 435327. https://bit.ly/30qoNRp

Astonitas, L. (2005). Personalidad, hábitos de consumo y riesgo de adicción al Internet en estudiantes universitarios. Revista de Psicología, 23(1), 65-112. http://revistas.pucp.edu.pe/index.php/psicologia/ article/view/981

Ato, M., López-García, J. J., \& Benavente, A. (2013). Un sistema de clasificación de los diseños de investigación en psicología. Anales de Psicología, 29(3), 10381059. http://dx.doi.org/10.6018/analesps.29.3.178511

Ávila-Toscano, J.H., Marenco-Escuderos, A., \& Madariaga, C. (2014). Indicadores bibliométricos, redes de coautorías y colaboración institucional en revistas colombianas de psicología. Avances en Psicología Latinoamericana, 32(1), 167-182. http://www.scielo.org.co/ pdf/apl/v32n1/v32n1a13.pdf

Becoña, E., \& Oblitas, L. A. (2003). Psicología de la salud y adicciones: perspectiva terapéutica. Revista de Psicología, 21(1), 71-106. https://doi.org/10.18800/ psico.200301.003

Bisso-Andrade, A. (2013). Adicción a internet: una amenaza contra la salud pública. Revista de la Sociedad Peruana de Medicina Interna, 26(2), 51-52. http://revistamedicinainterna.net/index.php/spmi/article/ view/328

Braun, A. C., De Lara, W., De Andrade, A. L., \& Ziebell , M. (2019). Why work-family conflict can drive your executives away? Revista de Psicología de la PUCP, 37(1), 251-278. https://doi.org/10.18800/psico.201901.009

Calle-Sarmiento, R., Tafur-Núñez, T., Ruiz-Huamán, K., \& Villar-Prado, S. (2013). Nivel de conocimiento sobre los efectos de los juegos online en los estudiantes del primer año de educación secundaria. Revista Enfermería Herediana, 6(2), 59-65. https://faenf.cayetano.edu.pe/images/pdf/Revistas/2013/febrero/ niveldeconocimientosobrelosefectosdelosjuegosonline.pdf

Cañón, S. C., Castaño, J. J., Hoyos, D. C., Jaramillo, J. C., Leal, D. R., Rincón, R., Sánchez, E.A., \& Urueña, L.S., (2016). Uso de internet y su relación con la salud de estudiantes universitarios de la ciudad de Man- zanales (Caldas Colombia), 1015-2016. Archivos de Medicina, 16(2), 312-325. https://www.redalyc.org/ pdf/2738/273849945010.pdf

Casale, S., \& Fioravanti, G. (2018). Why narcissists are at risk for developing Facebook addiction: The need to be admired and the need to belong. Addictive Behaviors, 76, 312-318. https://doi.org/10.1016/j.addbeh.2017.08.038

Castaño, J. J., \& Paez, M. L. (2019).Funcionalidad familiar y tendencias adictivas a internet y a sustancias psicoactivas en estudiantes universitarios. Psicología desde el Caribe, 36(2). https://www.redalyc.org/jatsRepo/213/21363396003/html/index.html

Castro, M., Celis, A., \& Medina, M. C. (2012). Uso de internet y niveles de dependencia entre estudiantes de Medicina Humana. Revista Horizonte Médico, 12(3), 25-32. https://www.horizontemedico.usmp.edu.pe/ index.php/horizontemed/article/view/128

Castro, S., \& Mahamud, K. (2017). Procrastinación académica y adicción a internet en universitarios de Lima Metropolitana. Avances en Psicología, 25(2), 189 197. http://revistas.unife.edu.pe/index.php/avancesenpsicologia/article/view/354

Challco, K., Rodríguez, S., \& Jaimes, J. (2016). Riesgo de adicción a redes sociales, autoestima y autocontrol en estudiantes de secundaria. Revista Científica de Ciencias de la Salud, 9(1), 9-15. https://doi. org/10.17162/rccs.v9i1.542

Chavarro, D., Ràfols, I., \& Tang, P. (2018). To what extent is inclusion in the Web of Science an indicator of journal 'quality'? Research Evaluation, 27(2), 106-118. https://doi.org/10.1093/reseval/rvy001

Chávez, J., Del Toro, M., \& López, O. (2017). Blog, Correo Electrónico y Foros Temáticos: Su Uso, Dominio y Actitud en Estudiantes de Educación Medio Superior de México. Hamut'ay, 4(2), 45-54. http://dx.doi. org/10.21503/hamu.v4i2.1471

Chen, C., \& Song, M. (2019). Visualizing a field of research: A methodology of systematic scientometric reviews. PloS one, 14(10), e0223994.

Cía, A.H. (2014). Las adicciones no relacionadas a sustancias (DSM-5, APA, 2013): un primer paso hacia la inclusión de las Adicciones Conductuales en las clasificaciones categoriales vigentes. Revista de Neuropsiquiatría, 76(4), 22-31. https://revistas.upch. edu.pe/index.php/RNP/article/view/1169

Clemente, L. A., Guzmán, I., \& Salas-Blas, E. (2018). Adicción a redes sociales e impulsividad en universitarios de Cusco. Revista de Psicología de Arequipa, 8(1), 15-37. https://revistas.ucsp.edu.pe/index.php/psicologia/ article/view/119/117

Contreras, A. J. (2015). Influencia de Facebook en las habilidades sociales de estudiantes universitarios de Chiclayo. Revista Científica Paian, 6(2). http://revistas. uss.edu.pe/index.php/PAIAN/article/view/240

Cotlear, I. Osada, J., Ceccarelli, M., Ruiz-Grosso, P., \& Vega-Dienstmaier, J. (2009). Sintomatología depresiva 
en usuarios de cabinas públicas de internet en un disttrito de Lima, Perú. Revista Peruana de Medicina Experimental y Salud Pública, 26(4), 462-465. http://www.scielo.org.pe/scielo.php?script=sci_arttext\&pid=S1726-46342009000400008

Cruzado, L., Matos, L., \& Kendall, R. (2006). Adicción a internet: Perfil clínico y epidemiológico de pacientes hospitalizados en un instituto nacional de salud mental. Revista Médica Herediana, 17(4), 196-205. http:// www.scielo.org.pe/pdf/rmh/v17n4/v17n4ao2.pdf

Curioso, W. H., \& Blas, M. M. (2007). Internet, adicción, cibersexo e infecciones de transmisión sexual en el Perú: ¿ Ficción o realidad?. Revista Medica Herediana, 18(1), 28-33. http://www.scielo.org.pe/scielo. php?pid=S1018-130X2007000100012\&script $=$ sci_arttext

Delgado, A.E., Escurra, M., Atalaya, M. C., Pequeña, J., Cuzcano, A., Rodríguez, R. E., \& Álvarez, D. (2016). Las habilidades sociales y el uso de redes sociales virtuales en estudiantes universitarios de Lima Metropolitana. Persona, 19, 55-75. https://revistas. ulima.edu.pe/index.php/Persona/article/download/971/932

Dhaha, I. S. Y. (2013). Predictors of Facebook addiction among youth: A Structural Equation Modeling (Sem). Journal of Social Sciences (COES\&RJ-JSS), 2(4), 186-195.

Domínguez, J. A., \& Ybañez, J. (2016). Adicción a las redes sociales y habilidades sociales en estudiantes de una institución educativa privada. Propósitos y representaciones, 4(2), 181-230. http://revistas.usil. edu.pe/index.php/pyr/article/view/122E

Donelly, E., \& Kuss, D.J. (2016). Depression among Users of Social Networking Sites (SNSs): The Role of SNS Addiction and Increased Usage. Journal of Addiction and Preventive Medicine, 1(2). http://irep.ntu.ac.uk/ id/eprint/29245/1/PubSub6778_Kuss.pdf

Echeburúa, E. (2012). Factores de riesgo y factores de protección en la adicción a las nuevas tecnologías y redes sociales en jóvenes y adolescentes. Revista española de drogodependencias, 37(4), 435-447. https://www. aesed.com/descargas/revistas/v37n4_5.pdf

Echeburúa, E. (Ed.) (2016). Abuso de internet ¿Antesala para la adicción al juego de azar online?. Pirámide. https://dialnet.unirioja.es/servlet/libro?codigo=656763

Escurra, M., \& Salas-Blas, E. (2014). Construcción y validación del Cuestionario de adicción a redes sociales (ARS). Liberabit, 20(1), 73-91. http://www.scielo.org. pe/pdf/liber/v20n1/a07v20n1.pdf

Espinoza, L. S. (2018). Trastornos de personalidad y juego patológico en adolescentes y jóvenes con dependencia de las máquinas tragamonedas. Persona, 21(2), 99-124. http://revistas.ulima.edu.pe/index. php/Persona/article/viewFile/3023/3091

Estévez, A., Urbiola, I. Iruarrizaga, I., Onaindia, J., \& Jauregui, P. (2017). Dependencia emocional en las rela- ciones de pareja y las consecuencias psicológicas de internet y el abuso mobil. Anales de psicología, 33(2), 260-268. https://revistas.um.es/analesps/article/view/analesps.33.2.255111/211001

Flores, C., Gamero, K., Aras, W. L., Melgar, C., Sota, A. \& Ceballos, K.D. (2015). Adicción al celular en estudiantes de la Universidad Nacional de San Agustín y la Universidad Católica San Pablo. Revista de Psicología (Universidad Católica San Pablo), 5(2), 13-25. https:// revistas.ucsp.edu.pe/index.php/psicologia/article/ view/141

Gamero, K., Flores, C., Arias, W. L., Ceballos, K.D., Román, A., \& Marquina, E. (2016). Estandarización del Test de Dependencia al Celular para estudiantes universitarios de Arequipa. Persona, 19, 179-200. https:// revistas.ulima.edu.pe/index.php/Persona/article/ viewFile/979/940

Goldberg, I. (1995). Internet Addiction disorder. Diadnostic criteria. http://users.rider.edu/ suler/psycyber/supportgp.html

Gómez-Burns, A., \& Arela-Bobadilla, R.W. (2017). Nativos digitales: dependencia de las redes sociales en internet y su relación con el desempeño académico. Educationis Momentum, 3(1), 107-143. https://revistas.ucsp.edu.pe/index.php/emomentum/article/ view/113

Han, D. H., Yoo, M., Renshaw, P. F. \& Petry, N. M. (2018). A cohort study of patients seeking Internet gaming disorder treatment. Journal of Behavioral Addictions 7(4), 930-938. https://doi.org/10.1556/2006.7.2018.102

Hernández, R., Fernández, C., \& Baptista-Lucio, M. P. (2014). Metodología de la investigación. Mcgraw-hill.

Hernández, R., \& Mendoza, C. P. (2018). Metodología de la investigación. Las rutas cuantitativa, cualitativa y mixta. McGraw-Hill Education.

Hormes, J. M., Kearns, B., y Timko, C. A. (2014). Craving Facebook? Behavioral addiction to online social networking and its association with emotion regulation deficits. Addiction, 109(12), 2079-2088. https://doi. org/10.1111/add.12713

Kuss, D. J., Griffiths, M. D., \& Pontes, H. M. (2017). Chaos and confusion in DSM-5 diagnosis of Internet Gaming Disorder: Issues, concerns, and recommendations for clarity in the field. Journal of Behavioral Addictions, 6(2), 103-109. https://doi. org/10.1556/2006.5.2016.062

Lam-Figueroa, N., Contreras-Pulache, H., Mori-Quispe, E., Nizama-Valladolid, M., Gutiérrez, C., Hinostroza-Camposano, W., Torrejón. E., Hinostroza-Camposanto, R., Coaquira-Condori, E., \& Hinostroza-Camposano, W.D. (2011). Adicción a internet: desarrollo y validación de un instrumento en escolares adolescentes de Lima, Perú. Revista Peruana de Medicina Experimental y Salud Publica, 28(3), 462-469. http:// www.scielo.org.pe/pdf/rins/v28n3/a09v28n3.pdf

Lara, K., \& Miyahira. J. (2009). Uso de Internet por estudiantes del internado de Medicina en Lima Metropolita- 
na. Revista Médica Herediana, 20(3), 156-161. http:// www.scielo.org.pe/pdf/rmh/v20n3/v20n3ao6.pdf

Leon, W. (2014). Adicción a internet en estudiantes de un instituto tecnológico. Hamut'ay, 1(2), 17-23. http:// revistas.uap.edu.pe/ojs/index.php/HAMUT/article/ view/694

Marín, C. (2018). Adicción a internet y funcionalidad familiar en universitarios de Lima Norte. Casus. Revista de investigación y casos en salud, 3(1), 1-8. http://casus.ucss.edu.pe/index.php/casus/article/ view/63/57

Martino, F. M. (2014). Las tecnologías de información y comunicación y el bienestar psicológico en la generación net. Hamut'ay, 1(1), 39-51. http://revistas.uap. edu.pe/ojs/index.php/HAMUT/article/view/572

Matalinares, M.L., Raymundo, O., \& Baca, R. (2014). Propiedades psicométricas del test de adicción al internet (TAI). Revista de Peruana de Psicología y Trabajo Social, 3(2), 45-66.

Matalinares, M.L., Díaz, G., Raymundo, O., Baca, D., Fernández, E., Uceda, J., Leyva, V., Sánchez, E., Villavicencio, N., Yaringaño, J., Torre, J., Encalada, M., \& Díaz, A. (2013b). Influencia de los estilos parentales en la adicción al internet en alumnos de secundaria del Perú. Revista de Investigación en Psicología, 16(2), 195 - 220. https://doi.org/10.15381/rinvp.v16i2.6554

Matalinares, M.L., Arenas, C. Díaz, G., Dioses, A., Yarlequé, L., Raymundo, O., Baca, D., Fernández, E., Uceda, J., Huari, Y., Villavicencio, N., Vargas, P., Quispe, M., Sánchez, E., Leyva, V., Díaz, A., Campos, A., \& Yaringaño, J. (2013a). Adicción a internet y agresividad en estudiantes de secundaria del Perú. Revista de investigación en psicología, 16(1), 75-93. http://revistasinvestigacion.unmsm.edu.pe/index.php/psico/article/viewFile/3920/3144

Mejía, G., Paz, J., \& Quinteros, D. (2014). Adicción a facebook y habilidades sociales en estudiantes de una universidad privada de Lima. Revista científica de ciencias de la salud, 7(1), 7-15. https://doi.org/10.17162/ rccs.v7i1.985

Morales, J. (2015). Uso de las tecnologías de información y comunicación en adolescentes de las instituciones educativas públicas del Distrito Mi Perú. Anales de la Facultad de Medicina, 76(3), 257-259. https://doi. org/10.15381/anales.v76i3.11235

Morales, L. F. (2016). Producción e impacto de las revistas peruanas del ámbito de las Ciencias Sociales en el catálogo Latindex. Investigación bibliotecológica, 30(69), 179-204. http://www.scielo.org.mx/scielo. php?pid=S0187-358X2016000200179\&script $=$ sci_arttext

Moreno-Guerrero, A. J., Gómez, G., López, J., \& Rodríguez, C. (2020). Internet addiction in the web of science database: a review of the literature with scientific mapping. International Journal of Environmental Research and Public Health, 17(8), 2753. https://doi. org/10.3390/ijerph17082753
Navas-Fernández, M., Abadal, E., \& Rodrigues, R.S. (2018). Internacionalidad de las revistas científicas españolas indexadas en Web of Science y Scopus. Revista Española de Documentación Científica, 41(3): e209. https://doi.org/10.3989/redc.2018.3.1498

Nizama-Valladolid, M. (2015). Innovación conceptual en adicciones (primera parte). Revista de Neuro-Psiquiatría, 78(1), 22-29. http://www.scielo.org.pe/scielo.php?script=sci_arttext\&pi d=S0034-85972015000100004

Nizama-Valladolid, M. (2015). Innovación conceptual en adicciones (segunda parte). Revista de Neuro-Psiquiatría, 78(2), 93-101. http://www.scielo.org. pe/scielo.php?pid=S0034-85972015000200006\&script=sci_arttext $\&$ tlng $=$ en

Nizama-Valladolid. M, Luna, F., \& Cachay, C. (2019). La adiccionología una especialidad médica y subespecialidad de la psiquiatría. Revista de la Facultad de Medicina Humana, 19(2), 89-99. http://revistas.urp.edu. pe/index.php/RFMH/article/view/2078

Oficina de las Naciones Unidas Contra la Droga y el Delito. (2019). El Informe Mundial sobre las Drogas 2019 hace un llamado a aumentar los recursos para ampliar la prevención y el tratamiento del consumo de drogas. https://cutt.ly/NgjK7Q5

Olmedo, I. J., Denis-Rodríguez, E., Barradas, M. E., Villegas, J. E., \& Denis, P. B. (2019). Agresividad y conducta antisocial en individuos con dependencia al teléfono móvil: un posible factor criminogénico. Horizonte Médico (Lima), 19(3), 12-19. http://www.scielo.org. pe/scielo.php?pid=S1727-558X2019000300003\&s cript $=$ sci_arttext

Padilla-Romero, C., \& Ortega-Blas, J. (2017). Adicción a las redes sociales y sintomatología depresiva en universitarios. CASUS. Revista de Investigación y Casos en Salud, 2(1), 47-53. http://casus.ucss.edu.pe/index. $\mathrm{php} / \mathrm{casus} /$ article/view/31

Palomares, R. (2017). Relaciones intrafamiliares y adicción a internet en estudiantes de secundaria de una institución educativa de villa maría del triunfo. Acta Psicológica Peruana, 2(1), 52- 65. http://revistas.autonoma.edu.pe/index.php/ACPP/article/view/68

Perestelo-Pérez, L. (2013). Standard son how to develop and report systematic reviews in Psychology and Health. International Journal of Clinical and Health Psychology, 13(1), 49-57. https://doi.org/10.1016/S16972600(13)70007-3

Rengifo, C. A., Mamani, L., \& Vilca, W. (2015). Funcionamiento familiar y adicción a internet en estudiantes de una institución educativa pública de Lima Norte. Revista Científica de Ciencias de la Salud, 8(2), 9-17. https://revistas.upeu.edu.pe/index.php/rc_salud/ article/view/245

Rojas-Jara, C. Polanco-Carrasco, R., Montenegro, C., Morales, C., Retamal, K., Rivano, N., \& Sepúlveda, J. (2020). Adicción a la comida: una revisión sobre el concepto, sus características y medición. Cuadernos 
de Neuropsicología/Panamerican Journal of Neuropsychology, 14(1), 142-151. http://www.cnps.cl/index.php/cnps/article/view/406

Rousseau, R. (2002). Journal evaluation: Technical and practical issues. Library Trends, 50(3), 418-439. https://www.ideals.illinois.edu/bitstream/handle/2142/8418/librarytrendsv50i3i_opt.pdf

Rücker, J., Akre, C., Berchtold, A., \& Suris, J. C. (2015). Problematic Internet use is associated with substance use in young adolescents. Acta Paediatrica, 104(5), 504507. https://doi.org/10.1111/apa.12971

Salas-Blas, E. (2014). Adicciones psicológicas y los nuevos problemas de salud. Cultura, 28, 111-146. http:// www.revistacultura.com.pe/revistas/RCU_28_1_ adicciones-psicologicas-y-los-nuevos-problemas-de-salud.pdf

Salas-Blas, E., \& Escurra, M. (2014). Uso de redes sociales entre estudiantes universitarios limeños. Revista Peruana de Psicología y trabajo Social, 3(1), 75-90.

Salas-Blas, E. (2019). Comprendiendo las limitaciones de la investigación [Número Especial]. Propósitos y Representaciones, 7, e424. http://dx.doi.org/10.20511/ pyr2019.v7nSPE.424

Salas-Blas, E., \& Copez-Lonzoy, A. (2018). ¡Necesito seguir trabajando!. Una revisión conceptual sobre la adicción al trabajo. Cultura, 32, 331-352. https://doi. org/10.24265/cultura.2018.v32.15

Salas-Blas, E., Merino-Soto, C., Chóliz, M., \& Marco, C. (2017). Análisis psicométrico del test de dependencia de videojuegos (TDV) en población peruana. Universitas Psychologica, 16(4), 1-13. https://doi.org/10.11144/ Javeriana.upsy16-4.aptd

Sanchez-Meca, J., \& Botella, J. (2010). Revisiones sistemáticas y meta-análisis: Herramientas para la práctica profesional. Papeles del Psicólogo, 31(1), 7-17. http:// www.papelesdelpsicologo.es/pdf/1792.pdf

Santillán-Aldana, J., Arakaki, M., de la Vega, A., Calderón-Carranza, M., \& Pacheco-Mendoza, J. (2017). Características generales de las revistas científicas peruanas. Revista Española de Documentación Científica, 40(3), e182. http://dx.doi.org/10.3989/ redc.2017.3.1419

Torres, R. J. (2015). Dimensiones de la personalidad y actitudes hacia el consumo de internet en jóvenes en consultoría privada. Hamut'ay, 2(2), 9-24. http://dx.doi. org/10.21503/hamu.v2i2.912
Uchuypoma, D. (2017). Juegos online: una mirada desde el juego patológico. Hamut'ay, 4(2), 55-64. http://dx. doi.org/10.21503/hamu.v4i2.1472

Valencia, D. I. (2016). Adicción a las redes sociales y hábitos de estudio en estudiantes de psicología. Acta Psicológica Peruana, 1(1), 36-47. http://revistas.autonoma.edu.pe/index.php/ACPP/article/view/44

Van Rooij, A., \& Prause, N. (2014). A critical review of internet addiction criteria with suggestions for the future. Journal of Behavioral Addictions, 3(4), 203-213. https://doi.org/10.1556/JBA.3.2014.4.1

Vara, R. (2017). Adicción a los videojuegos y agresividad en estudiantes de secundaria de dos colegios privados de Villa María del Triunfo. Acta Psicológica Peruana, 2(2), 193 - 216. http://revistas.autonoma.edu.pe/ index.php/ACPP/article/view/75

Vera-Villarroel, P., López-López, W., Lillo, S., \& Silva, L.M. (2010). La producción científica en psicología Latinoamericana: un análisis de la investigación por países. Revista Latinoamericana de Psicología, 43(1) 95-104. https://www.redalyc.org/ pdf/805/80520078009.pdf

Waltman, L., \& Van Eck, N. J. (2013). A smart local moving algorithm for large-scale modularity-based community detection. The European physical journal $B, 86(11), 471$.

Yarlequé, L. A., Javier, L., Núñez, E. R., Navarro, L. L., \& Matalinares, M. L. (2013). Internet y agresividad en estudiantes de secundaria del Perú. Horizonte de la Ciencia, 3(4), 103-110. https://www.redalyc.org/jatsRepo/5709/570960879015/570960879015.pdf

Yen, J-J. Lin, H-C., Chou, W-P., Liu, T-L., \& Ko, C-H. (2019). Associations Among Resilience, Stress, Depression, and Internet Gaming Disorder in Young Adults. International Journal Enviromental Research Public Health, 16(17), 3181. https://doi.org/10.3390/ijerph16173181

Young, K. S. (1998). Internet addiction: The emergence of a new clinical disorder. Cyber Psychology \& Behavior, 1(3), 237-244. https://doi.org/10.1089/cpb.1998.1.237

Yu, H., \& Cho, J. (2016). Prevalence of Internet Gaming Disorder among Korean Adolescents and Associations with Non-psychotic Psychological Symptoms, and Physical Aggression. American Journal of Health Behavior, 40(6), 705-716. https://doi.org/10.5993/ AJHB.40.6.3

RIDU / Revista Digital de Investigación en Docencia Universitaria / e-ISSN: 2223-2516

(c) Los autores. Este artículo es publicado por la Revista Digital de Investigación en Docencia Universitaria del Área de Institutional Research and Effectiveness de la Dirección de Aseguramiento de la Calidad, Universidad Peruana de Ciencias Aplicadas. . "Este es un artículo de acceso abierto, distribuido bajo los términos de la LicenciaCreativeCommons Atribución 4.0 Internacional (CC BY 4.0) (https://creativecommons.org/licenses/by/4.0/deed.es), que permite el uso, distribución y reproducción en cualquier medio, siempre que la obra original sea debidamente citada." 